\title{
Cardiovascular risk stratification in unselected primary care patients with newly detected arterial hypertension
}

\begin{abstract}
Thomas Dieterle', Joerg-Peter Sigle ${ }^{1}$, Gunar Bengel, Gerrit Kiefer, Verena Brenneisen and Benedict Martina
Cardiovascular risk (CVR) stratification in patients with arterial hypertension is essential. Few data are available on CVR factors (CVRFs), hypertensive target organ damage (TOD) and overall CVR in medical outpatients with newly detected arterial hypertension. General medical patients entering the Medical Outpatient Department of the University Hospital Basel, Switzerland, were screened for elevated office blood pressure (OBP of $>140 / 90 \mathrm{~mm} \mathrm{Hg}$ ). Patients with newly detected arterial hypertension (elevated OBP at two consultations) underwent a work-up that included fundoscopy, urinalysis, ambulatory blood pressure (ABP) monitoring, ECG and echocardiography. CVR was calculated according to the 1999/2003 World Health Organization/International Society of Hypertension (WHO/ISH) guidelines. A total of 2615 outpatients were screened. Of 580 patients with elevated first OPB, 207 were treated for hypertension, 98 refused to participate, 8 were early dropouts and 36 had a normal second OBP. Data from 212 patients were analyzed (mean age $53 \pm 14$ years). The first and second OBP readings were $162 \pm 6 / 100 \pm 6$ and $153 \pm 14 / 96 \pm 9 \mathrm{~mm} \mathrm{Hg}$, respectively. Mean ABP was $134 \pm 12 / 83 \pm 9 \mathrm{~mm} \mathrm{Hg}$, and sustained hypertension was found in $76.9 \%$ of patients. Among patients with hypertension according to OPB monitoring, $61.3 \%$ had 1 or 2 CVRFs, and $33.0 \%$ had $\geqslant 3$ CVRFs. Evidence of TOD, diabetes or associated clinical conditions (ACCs), such as renal or cardiovascular disease, was found in $26.4,5.6$ and $7.1 \%$ of patients, respectively. In terms of CVR, $2.4 \%$ of patients were at low risk, $25.9 \%$ at medium risk and $71.7 \%$ at high risk. No differences existed between white coat and sustained hypertensives regarding CVRFs, TOD or ACCs. Comprehensive analysis in patients with newly detected arterial hypertension revealed a surprisingly high prevalence of CVRFs, TOD and ACCs, indicating high CVR in the majority of these patients.
\end{abstract}

Hypertension Research (2010) 33, 607-615; doi:10.1038/hr.2010.40; published online 9 April 2010

Keywords: ambulatory blood pressure monitoring; arterial hypertension; risk stratification; left ventricular hypertrophy; microalbuminuria

\section{INTRODUCTION}

The aim of antihypertensive treatment is to prevent adverse cardiovascular events. As the absolute benefit of treatment depends mainly on pretreatment cardiovascular risk (CVR), it is the main determinant for the decision to start blood pressure-lowering therapy in patients with hypertension. ${ }^{1,2}$ The 1999 World Health Organization/International Society of Hypertension (WHO/ISH) guidelines for the management of hypertension included for the first time individual risk stratification in the antihypertensive treatment schedule. ${ }^{3}$ According to these guidelines, immediate drug therapy for grade 1 and 2 hypertension is recommended only for patients with an estimated risk of cardiovascular events above $20 \%$ over the next 10 years. Grade 1 and 2 hypertensives at lower risk may be monitored for up to 1 year without blood pressure-lowering therapy.

The WHO/ISH guidelines for the management of arterial hypertension define high-risk patients who will require immediate antihypertensive therapy as having the office blood pressure (OBP) values of $\geqslant 180 / 110 \mathrm{~mm} \mathrm{Hg}, \geqslant 3$ cardiovascular risk factors (CVRFs), evidence of target organ damage (TOD) such as microalbuminuria, left ventricular hypertrophy $(\mathrm{LVH})$ or hypertensive retinopathy grade III/IV, or evidence of associated clinical conditions (ACCs) such as diabetes mellitus or cardiac, cerebral or renal disease. ${ }^{1,3}$ Microalbuminuria and LVH are common findings in essential hypertension and are, in addition, independent CVRFs. ${ }^{4,5}$

Estimates of the prevalence of $\mathrm{LVH}$ in essential hypertension range from 12 to $70 \%$ depending largely on the measurement technique used. ${ }^{6-10}$ Standard ECG has a low sensitivity to detect increased left ventricular mass, despite the use of a variety of criteria. ${ }^{11,12}$ Nevertheless, the use of echocardiography is not recommended as a standard procedure in patients with newly detected hypertension, mainly because of limited availability and increased cost. Testing for microalbuminuria, although an established marker of hypertensive organ damage, ${ }^{13-15}$ is not yet routinely performed in clinical practice, ${ }^{4}$ and its usefulness has even been challenged recently. ${ }^{16}$

Detection of microalbuminuria or LVH affects therapeutic decisions mainly in patients at low or moderate risk, because immediate drug 
treatment is indicated under these circumstances. Previous studies showed that echocardiography modifies the risk classification in up to $29 \%$ of patients, therefore identifying a considerable number of patients requiring immediate drug treatment. ${ }^{17-19}$ A similar effect on risk stratification was described when data on microalbuminuria were applied in the risk stratification of hypertensive patients. ${ }^{20}$

However, most of these studies used elevated OBP for the definition of hypertension. Ambulatory blood pressure (ABP) monitoring is a very useful tool for improving risk stratification in patients with hypertension. $^{21,22}$ In fact, $\mathrm{ABP}$ was found to be correlated more strongly with TOD score than was OBP. ${ }^{23}$

Furthermore, these studies were performed in selected patient populations with a previously established diagnosis of arterial hypertension. Very few data are available on the distribution of blood pressure, the prevalence of CVRFs, TOD and ACCs in an unselected general medical outpatient population with newly detected arterial hypertension. It is not clear whether risk stratification yields similar results in a group of unselected patients with definite hypertension and to what extent the individual parameters contribute to risk stratification.

Therefore, the aim of this study was to assess the current distribution of office and ambulatory blood pressure as well as CVRFs, TOD and ACCs in a general medical outpatient population admitted over 1 year to a medical outpatient department. An additional aim was to analyze the effect of the currently recommended diagnostic approach on CVR stratification and the contribution of consecutive diagnostic steps, especially the value of echocardiography and microalbuminuria, for risk stratification in unselected primary care patients with newly detected arterial hypertension.

\section{METHODS}

\section{Setting}

The study was conducted in the Medical Outpatient Department of the University Hospital Basel, Switzerland. This department provides primary care for general medical patients. Most are treated as walk-in patients, and approximately $20 \%$ are referred by general practitioners for a second opinion or for interdisciplinary ambulatory care. All patients are routinely seen by third- or fourth-year residents, who are supervised by an attending physician board certified in Internal Medicine.

\section{Study population}

All adult general medical patients entering the Medical Outpatient Department of the University Hospital Basel between April 2001 and March 2002 were screened for elevated OBP values by a hypertension clinic nurse. A minimum of two blood pressure readings were obtained, with the patient in a sitting position after at least $5 \mathrm{~min}$ of rest in a quiet environment, using appropriately sized blood pressure cuffs. Patients with elevated OBP at the first visit underwent further clinical work-up.

Patients referred to the outpatient hypertension clinic were considered to be preselected and were not included. Further exclusion criteria were pretreated hypertension, treatment with blood pressure-lowering medication for reasons other than hypertension, absence of informed consent, severe concomitant illness with the exception of diabetes mellitus, and compelling indications for echocardiography, for example, suspected pericarditis, heart failure or heart murmur suggesting valvular heart disease. The study protocol was approved by the local ethical committee, and all participants gave written informed consent.

\section{Grading of OBP}

Elevated OBP was defined as systolic blood pressure of $\geqslant 140 \mathrm{~mm} \mathrm{Hg}$ or diastolic blood pressure of $\geqslant 90 \mathrm{~mm} \mathrm{Hg}$. Hypertension was defined as systolic OBP measurements of $\geqslant 140 \mathrm{~mm} \mathrm{Hg}$ or diastolic OBP measurements of $\geqslant 90 \mathrm{~mm} \mathrm{Hg}$ at two consecutive visits. OBP was graded according to the 1999/2003 WHO/ISH guidelines, ${ }^{1,3}$ and was based on the values obtained at the second office visit. Grade 1 was defined as systolic blood pressure of 140-159 mm Hg or diastolic blood pressure of 90-99 mm Hg; grade 2 was defined as systolic blood pressure of $160-179 \mathrm{~mm} \mathrm{Hg}$ or diastolic blood pressure of $100-109 \mathrm{~mm} \mathrm{Hg}$ and grade 3 was defined as systolic blood pressure of $\geqslant 180 \mathrm{~mm} \mathrm{Hg}$ or diastolic blood pressure of $\geqslant 110 \mathrm{~mm} \mathrm{Hg}$.

\section{ABP measurement}

In addition to standard procedures, 24-h $\mathrm{ABP}$ monitoring using validated devices (Spacelabs, Switzerland, and Mobilograph, Stolberg, Germany) was performed in all patients after the second visit when OBP at this visit exceeded $140 \mathrm{~mm} \mathrm{Hg}$ (systolic) or $90 \mathrm{~mm} \mathrm{Hg}$ (diastolic). Normal ABP monitoring values were defined as a mean daytime $\mathrm{ABP}$ of $<135 / 85 \mathrm{~mm} \mathrm{Hg}$.

\section{Grading of CVR}

CVR was graded according to the $2003 \mathrm{WHO} / \mathrm{ISH}$ guidelines. ${ }^{1,3}$ Low risk was defined as grade 1 hypertension in the absence of diabetes, clinical cardiovascular disease, TOD and CVRFs. Patients were classified as being at medium risk if they had grade 1 hypertension with one or two risk factors or grade 2 hypertension with fewer than three risk factors in the absence of TOD. High or very high risk was defined as having grade 3 hypertension with at least three CVRFs, or TOD, clinical cardiovascular disease or diabetes. ${ }^{1,3}$

\section{Procedures used for risk stratification}

Patients with newly detected office hypertension received routine clinical workups according to 1999 and $2003 \mathrm{WHO} /$ ISH guidelines; ${ }^{1,3}$ that is, taking the past medical history and a complete physical examination, including fundoscopy, routine blood cell count and blood chemistry tests, a standard 12-lead ECG and urinalysis.

Variables for risk stratification included CVRFs such as age, gender, smoking status, family history with respect to premature cardiovascular disease, total cholesterol levels ( $>6.5 \mathrm{mmoll}^{-1}$ was considered elevated), body mass index and level of physical activity. Physical inactivity was defined as no physical activity or physical activity on a less than weekly basis. TOD was considered when microalbuminuria was present upon urinalysis, when evidence of LVH was found in the standard 12-lead ECG or when hypertensive retinopathy grade III or IV was detected by fundoscopy. ACCs were considered with a positive history or clinical signs and symptoms of diabetes mellitus or cardiovascular, renal, cerebrovascular or peripheral vascular disease.

In addition, all patients underwent echocardiography for quantification of left ventricular function and mass.

CVR was calculated from these parameters according to the 1999 and 2003 WHO/ISH guidelines. ${ }^{1,3}$

\section{Screening for TOD}

Microalbuminuria. Renal TOD was evaluated using microalbuminuria; that is, an albumin excretion that exceeds the normal range $\left(>20 \mathrm{mgl}^{-1}\right)$ but is below the minimum level of detection by standard urinary dipsticks (usually $<200$ to $300 \mathrm{mgl}^{-1}$ ). Microalbuminuria was assessed using quantitative assays to measure the albumin/creatinine ratio. Creatinine concentrations in urine specimens were measured using enzymatic methods (Wako, Pure Chemical Industries, Osaka, Japan) with a chemistry analyzer (Hitachi 917, Tokyo, Japan). Albumin concentrations in urine specimens were determined using nephelometric methods (Beckman Coulter, Brea, CA, USA). Microalbuminuria, as a marker for renal TOD, was defined as an albumin/creatinine ratio of $>2.26 \mathrm{mg} \mathrm{mmol}^{-1}$ in a clean midstream sample. ${ }^{24}$

Left ventricular hypertrophy. Electrocardiography: Standard 12-lead ECG was performed in all patients upon entry into the study. The Sokolow-Lyon Index ${ }^{25}$ and Cornell voltage product were calculated using the following formulas ${ }^{26}$ :

$$
\begin{aligned}
& \text { Sokolow-Lyon index: } \mathrm{S}_{\mathrm{V} 1}(\mathrm{mV})+\mathrm{R}_{\mathrm{V} 5}(\mathrm{mV}) \\
& \text { Cornell voltage product: } \left.\left[\mathrm{R}_{\mathrm{AVL}}(\mathrm{mV})+\mathrm{S}_{\mathrm{V} 3}(\mathrm{mV})+8 \text { (in women }\right)\right] \times \mathrm{QRS}(\mathrm{ms})
\end{aligned}
$$

Electrocardiographic LVH was defined as a Sokolow-Lyon Index of $>3.5 \mathrm{mV}$ or Cornell voltage product of $>2440 \mathrm{~mm} \times \mathrm{ms}^{25,26}$ 
Echocardiography: All patients underwent transthoracic echocardiography using a Philips Sonos 5500 ultrasound system (Philips Switzerland, Zurich, Switzerland). Echocardiographic exams were performed by one of two experienced cardiologists and were stored on magneto-optical disks for offline analysis.

Dimensions of interventricular septum thickness, posterior wall thickness and end-diastolic diameter were measured according to the guidelines of the American Society of Echocardiography ${ }^{27}$ by one of two experienced cardiologists blinded to patient name and clinical data. Left ventricular mass (LVM) was calculated according to the following formula: ${ }^{28}$

$$
\left.\operatorname{LVM}(\mathrm{g})=0.8 \times\left(1.04 \times(\text { IVSth }+ \text { PWth }+\mathrm{EDD})^{3}-\mathrm{EDD}^{3}\right)+0.6\right)
$$

where IVS is interventricular septum thickness, PW is posterior wall and EDD is end-diastolic diameter.

LVM was normalized to body surface area (LVM index), and echocardiographic LVH was defined as a LVM index of $>134 \mathrm{~g} \mathrm{~m}^{-2}$ for men and $>110 \mathrm{~g} \mathrm{~m}^{-2}$ for women. ${ }^{28}$ Great care was taken to achieve optimal tracing quality.

\section{Statistics}

Standard descriptive analyses were undertaken. Data are presented as means \pm s.d. unless otherwise stated.

According to estimated future CVR, study patients were classified into three categories: low-risk, medium-risk or high-risk patients. ${ }^{1,3}$ First, medical history, including CVRFs and the results of the ECG and other clinical and routine laboratory exams, were considered for purposes of risk stratification. Then, the presence or absence of microalbuminuria or echocardiographic LVH was added, and the proportional contribution of each parameter to the risk stratification model was calculated. Finally, risk stratification was calculated including all available data.

Comparisons between patients with sustained and white coat hypertension were performed using the Mann-Whitney $U$-test or independent samples $t$-test where applicable. Distribution of risk factors and overall CVR were compared between groups using the Kolmogorov-Smirnov test.

A $P$-value of $<0.05$ was considered to be statistically significant. Statistical analyses was performed using Microsoft Excel 2002 (Microsoft, Redmond, WA, USA) and SPSS for Windows version 11.0 (SPSS, Chicago, IL, USA).

\section{RESULTS}

\section{Screening}

From April 2001 to March 2002, 2615 consecutive unselected medical outpatients entering the Medical Outpatient Department of the
University Hospital Basel were screened for elevated blood pressure values (Figure 1). Elevated OBP values were found in 580 patients. Of these, 207 patients had treated hypertension, 98 patients (for example, migrants) refused participation, 5 patients were early dropouts and 36 patients had normal OBP values at their second visit. This last patient group was separately analyzed.

In 19 patients, data required for risk stratification were missing because of incomplete work-up, insufficient echocardiographic image quality or missing urine samples. Thus, 212 patients were included in the analysis.

\section{Patient characteristics}

Office and ambulatory blood pressure. Baseline characteristics of the study population are shown in Table 1. Mean age was 53.2 years, ranging from 25 to 82 years. Mean OBP was $162 \pm 6 / 100 \pm 6 \mathrm{~mm} \mathrm{Hg}$ at visit 1 and $153 \pm 14 / 96 \pm 9 \mathrm{~mm} \mathrm{Hg}$ at visit 2 .

Mean ABP in all patients was $134 \pm 12 / 83 \pm 9 \mathrm{~mm} \mathrm{Hg}$. Sustained hypertension, defined as daytime $\mathrm{ABP}$ of $\geqslant 135 / 85 \mathrm{~mm} \mathrm{Hg}$, was found in 163 of 212 patients (76.9\%). Mean $\mathrm{ABP}$ in these patients was $138 \pm 10 / 86 \pm 6 \mathrm{~mm} \mathrm{Hg}$ compared with $121 \pm 7 / 73 \pm 6 \mathrm{~mm} \mathrm{Hg}$ in the remaining 49 patients classified as white coat hypertensives.

As shown in Table 2, no significant difference was found for systolic OBP in patients with white coat hypertension when compared with sustained hypertension $(P=0.097)$, whereas diastolic OBP was significantly higher in sustained hypertension $(P=0.005)$.

According to the OBP measurements, $17.5 \%$ of all patients had stage 1 hypertension, $65.0 \%$ had stage 2 and $17.5 \%$ had stage 3 hypertension. Isolated systolic hypertension was found in $18.4 \%$ of the patients.

Although more patients with sustained hypertension in ABP measurements presented with stage 3 hypertension, the distribution of blood pressure stages did not differ significantly between patients with sustained and white coat hypertension.

Cardiovascular risk factors. The most prevalent CVRFs were physical inactivity, followed by positive family history for cardiovascular disease, smoking and hyperlipidemia. In the majority of patients (61.3\%), 1 or 2 CVRFs were present. Approximately one-third of

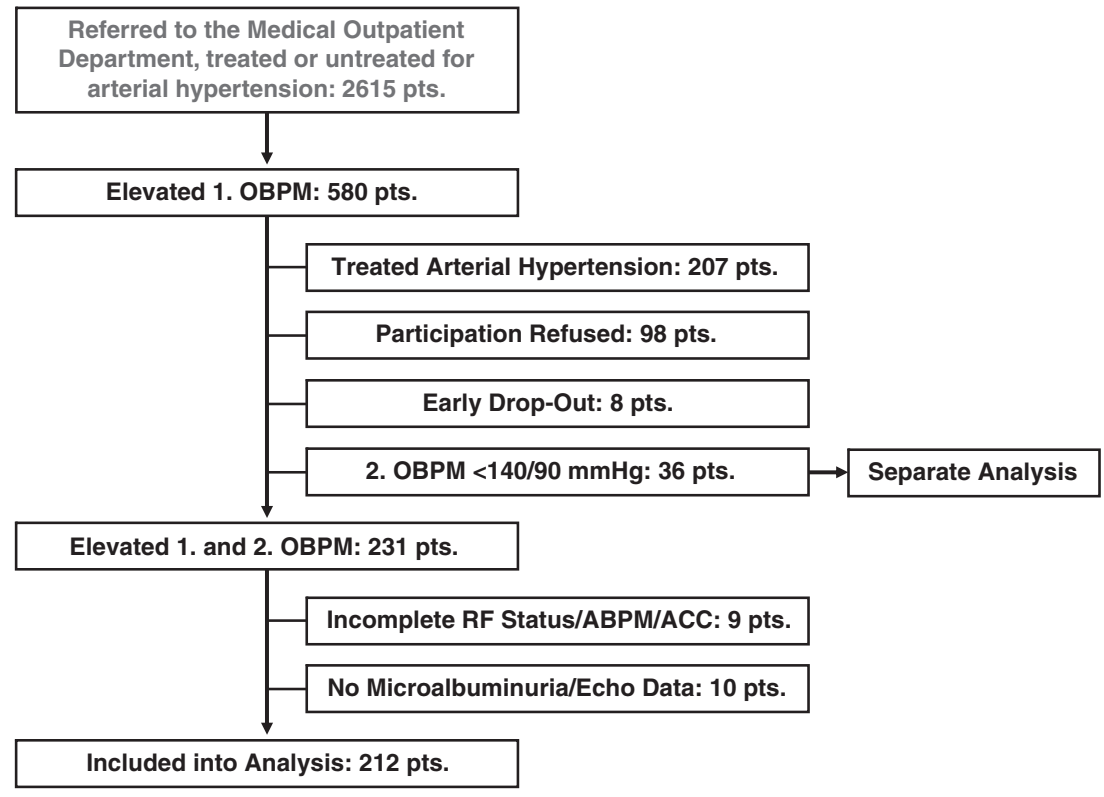

Figure 1 Patient flow. 
Table 1 Baseline characteristics of patients with elevated office blood pressure at two consecutive visits

\begin{tabular}{|c|c|}
\hline Parameter & \\
\hline$n$ & 212 \\
\hline Age (years, range) & $53.2 \pm 13.5(25-82)$ \\
\hline Sex (female/male, $n$ ) & $42.5 \%(90) / 57.5 \%(122)$ \\
\hline Body mass index $\left(\mathrm{kg} \mathrm{m}^{-2}\right)$ & $27.1 \pm 4.9$ \\
\hline \multicolumn{2}{|l|}{ Office blood pressure ( $\mathrm{mm} \mathrm{Hg}$ ) } \\
\hline Visit 1 (SBP/DBP) & $162 \pm 6 / 100 \pm 6$ \\
\hline Visit 2 (SBP/DBP) & $153 \pm 14 / 96 \pm 9$ \\
\hline Blood pressure stage $(1 / 2 / 3)$ & $17.5 / 65 / 17.5 \%$ \\
\hline \multicolumn{2}{|l|}{ Ambulatory blood pressure ( $\mathrm{mm} \mathrm{Hg}$ ) } \\
\hline $24 \mathrm{~h}(\mathrm{SBP} / \mathrm{DBP})$ & $134 \pm 12 / 83 \pm 9$ \\
\hline Day (SBP/DBP) & $139 \pm 12 / 87 \pm 9$ \\
\hline Night (SBP/DBP) & $126 \pm 14 / 76 \pm 9$ \\
\hline White coat hypertension & $23.1 \%$ \\
\hline \multicolumn{2}{|l|}{ Risk factors } \\
\hline Smoking & $38.2 \%$ \\
\hline Dyslipidemia & $23.6 \%$ \\
\hline Obesity & $21.7 \%$ \\
\hline Physical inactivity & $48.6 \%$ \\
\hline Family history of CV disease & $40.1 \%$ \\
\hline Risk factor count $(0 / 1-2 / \geqslant 3)$ & $5.7 / 61.3 / 33.0 \%$ \\
\hline \multicolumn{2}{|l|}{ Target organ damage } \\
\hline \multicolumn{2}{|l|}{ Echo parameters } \\
\hline Left ventricular mass (g) & $207 \pm 59$ \\
\hline Cardiac mass index $\left(\mathrm{g} \mathrm{m}^{-2}\right)$ & $111 \pm 28$ \\
\hline Echocardiographic LVH & $28.3 \%$ \\
\hline E/A ratio & $1.08 \pm 0.3$ \\
\hline \multicolumn{2}{|l|}{ ECG parameters } \\
\hline Sokolow-Lyon index (>3.5 mV) & $9.4 \%$ \\
\hline Cornell product ( $>2440 \mathrm{mV} \times \mathrm{ms}$ ) & $16.5 \%$ \\
\hline Electrocardiographic LVH & $21.2 \%$ \\
\hline Microalbuminuria & $19.3 \%$ \\
\hline Hypertensive retinopathy III/IV & $3.9 \%$ \\
\hline \multicolumn{2}{|l|}{ Associated clinical conditions } \\
\hline Diabetes mellitus & $5.6 \%$ \\
\hline Evidence of CV or renal disease & $7.1 \%$ \\
\hline
\end{tabular}

Abbreviations: $\mathrm{CV}$, cardiovascular; DBP, diastolic blood pressure $(\mathrm{mm} \mathrm{Hg})$; $\mathrm{LVH}$, left ventricular hypertrophy; SBP, systolic blood pressure $(\mathrm{mm} \mathrm{Hg})$.

patients presented with three of more risk factors. No significant differences between patients with white coat and sustained hypertension were found with respect to the number and distribution of CVRFs.

Microalbuminuria. Microalbuminuria, defined as an albumin/creatinine ratio of $\geqslant 2.26 \mathrm{mg} \mathrm{mmol}^{-1}$, was found in $19.3 \%$ of all patients. When compared with patients with sustained hypertension, patients with white coat hypertension tended to have microalbuminuria less frequently $(P=0.107)$.

Left ventricular hypertrophy. Electrocardiographic LVH was present in $21.2 \%$ of all patients, whereas echocardiography detected LVH in $28.3 \%$ of all patients. Cardiac mass index was $112 \pm 28 \mathrm{~g} \mathrm{~m}^{-2}$ in patients with definite hypertension and $107 \pm 27 \mathrm{~g} \mathrm{~m}^{-2}$ in those with white coat hypertension. Neither LVM and LVM index nor the prevalence of echocardiographic or electrocardiographic LVH differed significantly between sustained and white coat hypertensives.

Microalbuminuria and LVH. Evidence of both microalbuminuria and echocardiographic LVH was found in 13 of 212 patients (6.1\%), and microalbuminuria and electrocardiographic LVH in 12 of 212 patients $(5.7 \%)$. In addition, 16 of 212 patients $(7.5 \%)$ showed evidence of both electrocardiographic and echocardiographic LVH. In 5 of 212 patients $(2.4 \%)$, combined microalbuminuria, electrocardiographic and echocardiographic LVHs were present.

Grade III/IV hypertensive retinopathy. Hypertensive retinopathy was present in $3.9 \%$ of all patients, with the percentage of patients showing hypertensive retinopathy being slightly higher $(4.3 \%)$ in sustained than in white coat hypertension.

\section{Stratification of CVR}

Routine clinical evaluation. The results of individual risk stratification in all patients after routine clinical work-up are shown in Figures 2 and 3. On the basis of information from medical histories, clinical examinations, laboratory investigations and ECGs, $2.4 \%$ of patients were classified as being at low, $38.2 \%$ at medium and $59.4 \%$ at high CVR. No difference in the distribution of risk categories was found between patients with sustained and white coat hypertension.

Addition of microalbuminuria. The inclusion of data on microalbuminuria increased the percentage of patients at high CVR to $66.0 \%$. In this analysis, $2.4 \%$ of patients remained at low and $31.6 \%$ of patients remained at medium CVR. No differences were found in the distribution of risk between patients with sustained and white coat hypertension. The inclusion of microalbuminuria detected an additional 14 high-risk patients who would otherwise have been classified as being at medium CVR.

Addition of echocardiographic data. When compared with 'conventional' risk stratification, the inclusion of echocardiographic data increased the percentage of patients at high CVR to $67.4 \%$. In addition, $2.4 \%$ of patients were at low and $30.2 \%$ of patients at medium CVR. Again, no differences were found between patients with sustained and white coat hypertension regarding the distribution of risk. The inclusion of echocardiographic data identified an additional 17 high-risk patients who would otherwise have been classified as being at medium CVR.

Addition of microalbuminuria and echocardiographic data. When compared with 'conventional' risk stratification, the inclusion of both microalbuminuria and echocardiographic data highly significantly increased the percentage of patients at high CVR to $71.7 \%$. $(P=0.0011)$, whereas 2.4 and $25.9 \%$ of patients remained at low and medium CVR, respectively. No differences were found between patients with sustained and white coat hypertension regarding the distribution of risk. When compared with conventional risk stratification, the addition of both diagnostic modalities detected an additional 26 patients at high risk for cardiovascular events who would otherwise have been classified as being at medium CVR.

\section{Contribution of elevated OBP, CVRFs or signs of TOD to the classification of high CVR}

The contribution of elevated OBP, CVRFs, signs of TOD or ACCs to the classification of being at high CVR is given in Table 3 . 
Table 2 Characteristics of patients with sustained and white coat hypertension as defined by ambulatory blood pressure measurements

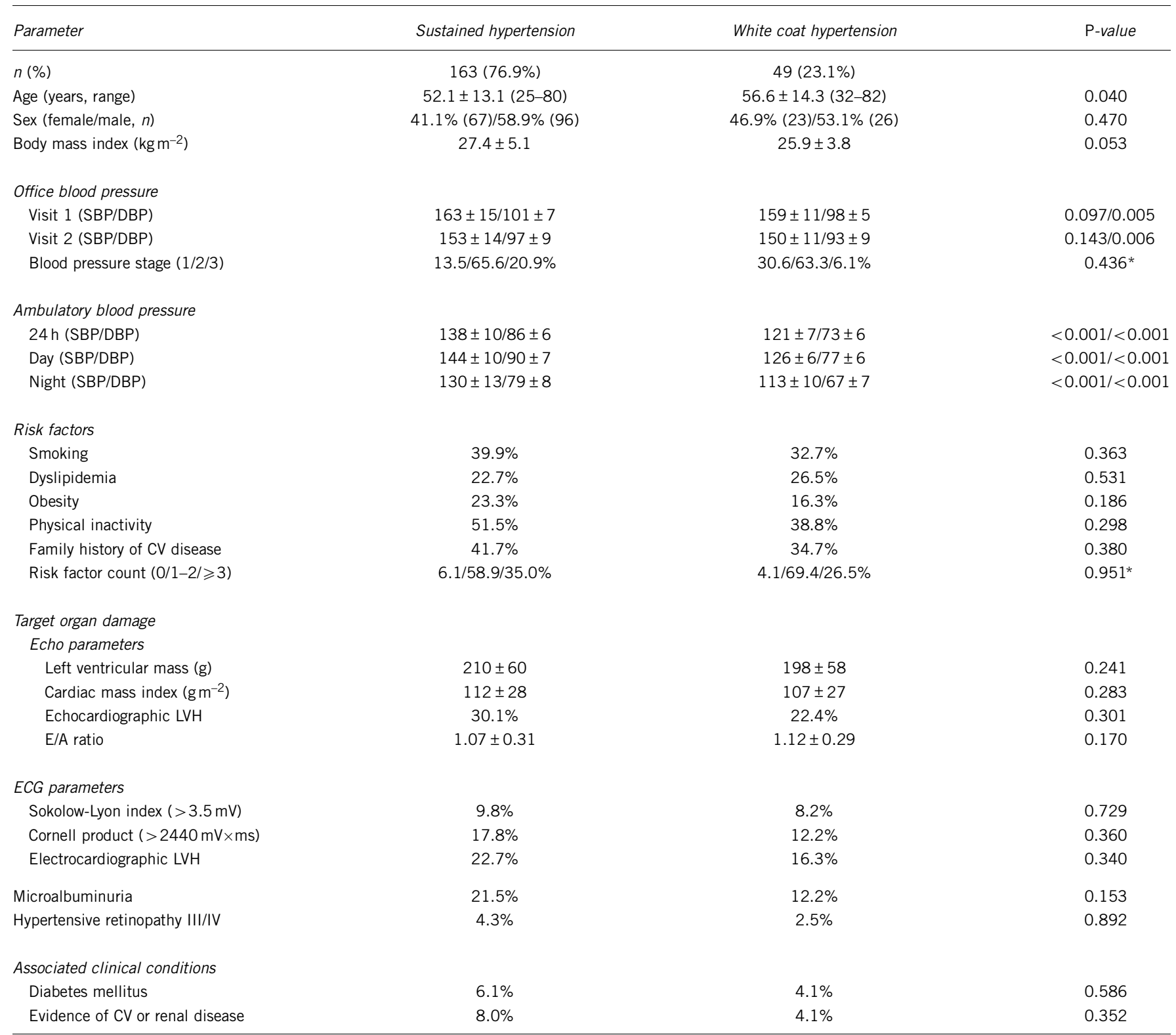

Abbreviations: CV, cardiovascular; DBP, diastolic blood pressure $(\mathrm{mm} \mathrm{Hg})$; LVH, left ventricular hypertrophy; SBP, systolic blood pressure ( $\mathrm{mm} \mathrm{Hg}$ ).

* $P$-value for comparison of distribution.

The majority of all patients (59.4\%) presented with either elevated OBP stage 3 or three or more CVRFs or evidence of TOD.

In six patients $(2.8 \%)$, elevated OBP alone predicted a high CVR. The cluster of three or more CVRFs predicted high CVR in $11.3 \%$ of patients, irrespective of the grade of blood pressure elevation. Similarly, the presence of TOD alone led to classification of high CVR in another $26.4 \%$ of the patients. In addition, $1.9 \%$ of patients were classified as being at high CVR solely on the basis of evidence of ACCs.

Risk stratification in white coat hypertension

Standard CVR stratification, including past medical history, clinical assessment, laboratory investigations and ECG, was performed in patients with white coat hypertension. Accordingly, $2.0 \%$ of these patients were classified as being at low, $49.0 \%$ at medium and $49.0 \%$ at high CVR.
With comparable rates of TOD in patients with white coat and sustained hypertension, risk stratification after addition of data regarding microalbuminuria and echocardiographic LVH did not differ significantly between the two groups. When both criteria for TOD were included in the stratification analysis, $2.0 \%$ of white coat hypertensives were classified as being at low, $36.7 \%$ at medium and $61.2 \%$ at high CVR.

There was no difference between white coat and sustained hypertension regarding the proportion of patients being at low, medium and high CVR.

Predictors for LVH in low- and medium-risk hypertensive patients In patients classified as being at low or medium risk after stratification, including microalbuminuria status, univariate analysis was performed to define factors associated with echocardiographic LVH and 


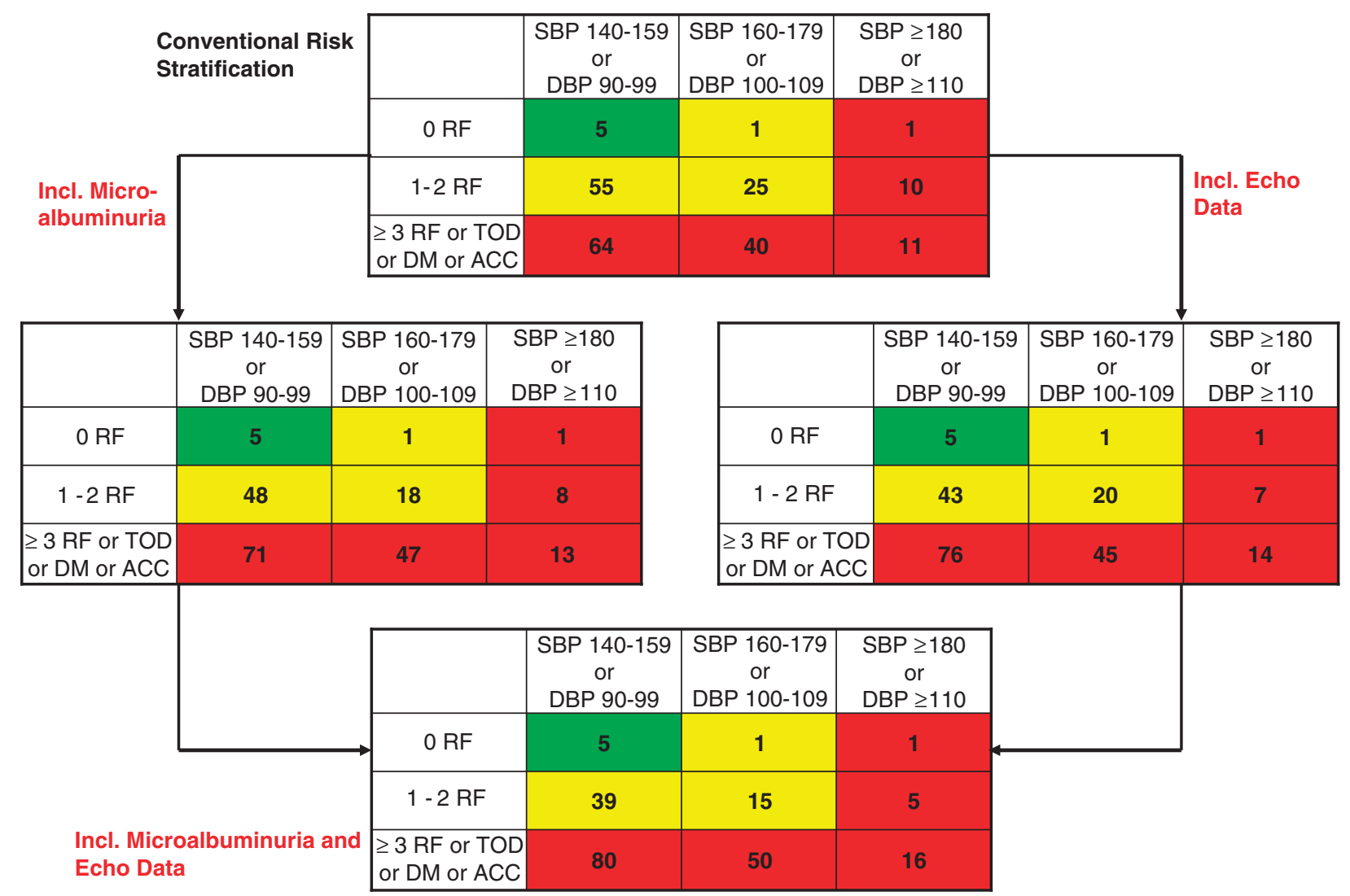

Figure 2 Distribution of office blood pressure, cardiovascular risk factors, evidence of target organ damage and associated clinical conditions in patients with elevated office blood pressure at two consecutive visits. ACC, associated clinical condition; DM, diabetes mellitus; DBP, diastolic blood pressure (mm Hg); $\mathrm{RF}$, cardiovascular risk factor; SBP, systolic blood pressure $(\mathrm{mm} \mathrm{Hg})$; TOD, target organ damage.

high CVR. Factors associated with echocardiographic LVH were age, body mass index, OBP, score on the Sokolow-Lyon index, smoking status, level of physical activity and family history with respect to CVR.

\section{Hypertension in patients with normal second OBP}

The characteristics of patients with normal OBP at the second visit are listed in Table 4. Patients in this group were younger and had lower $\mathrm{ABP}$ values when compared with patients with elevated OBP at both the first and second visits. Nevertheless, one-third of these patients were diagnosed to be hypertensive in the subsequent $\mathrm{ABP}$ measurement. The number and distribution of risk factors and TOD did not seem to be different when compared with patients with elevated OBP at both the first and second visits.

\section{DISCUSSION}

Arterial hypertension remains the most important risk factor for cardiovascular diseases. Decisions about treatment and optimal therapy for an individual patient should be based on overall CVR, the presence of hypertensive TOD and clinical conditions such as diabetes mellitus and cardio- or cerebrovascular disease. Therefore, current guidelines emphasize the importance of CVR stratification in patients with arterial hypertension. ${ }^{1}$ Few data are available regarding the CVR in an unselected general medical outpatient population. Moreover, little is known about the value of various diagnostic steps recommended for CVR stratification in an individual patient. The aim of this study was to define the overall CVR in an unselected medical outpatient population with newly detected arterial hypertension. An additional aim was to define the value of different diagnostic tools for risk stratification, especially the value of urinalysis for the detection of microalbuminuria, echocardiography and ABP measurement to develop an optimal stepwise approach to diagnosing patients with arterial hypertension.

Over a 1-year period, 2615 unselected consecutive general medical outpatients were screened for elevated OBP. Newly detected arterial hypertension, defined according to standard guidelines, ${ }^{3}$ was found in $8.8 \%$ of the screened population, with the majority of patients presenting with mild-to-moderate blood pressure elevation. Approximately $60 \%$ of patients presented with one or two CVRFs, and $33 \%$ of patients had three or more such factors. Despite only mild-tomoderate blood pressure elevation in the majority of patients, a substantial prevalence of TOD was found, with electrocardiographic or echocardiographic LVH being present in 21.2 and $28.3 \%$ of patients, respectively, and microalbuminuria in $19.3 \%$ of patients. Interestingly, only about one in six patients had evidence of combined TOD, and the combination of electrocardiographic and echocardiographic LVH was present in only $17.9 \%$ of patients. The high prevalence of CVRFs and TOD is reflected by a high CVR in this unselected medical outpatient population with newly detected arterial hypertension. Taking into account all diagnostic modalities used in our study, $>70 \%$ of patients were classified as being at high CVR, and only $2.4 \%$ of patients had a low CVR. 

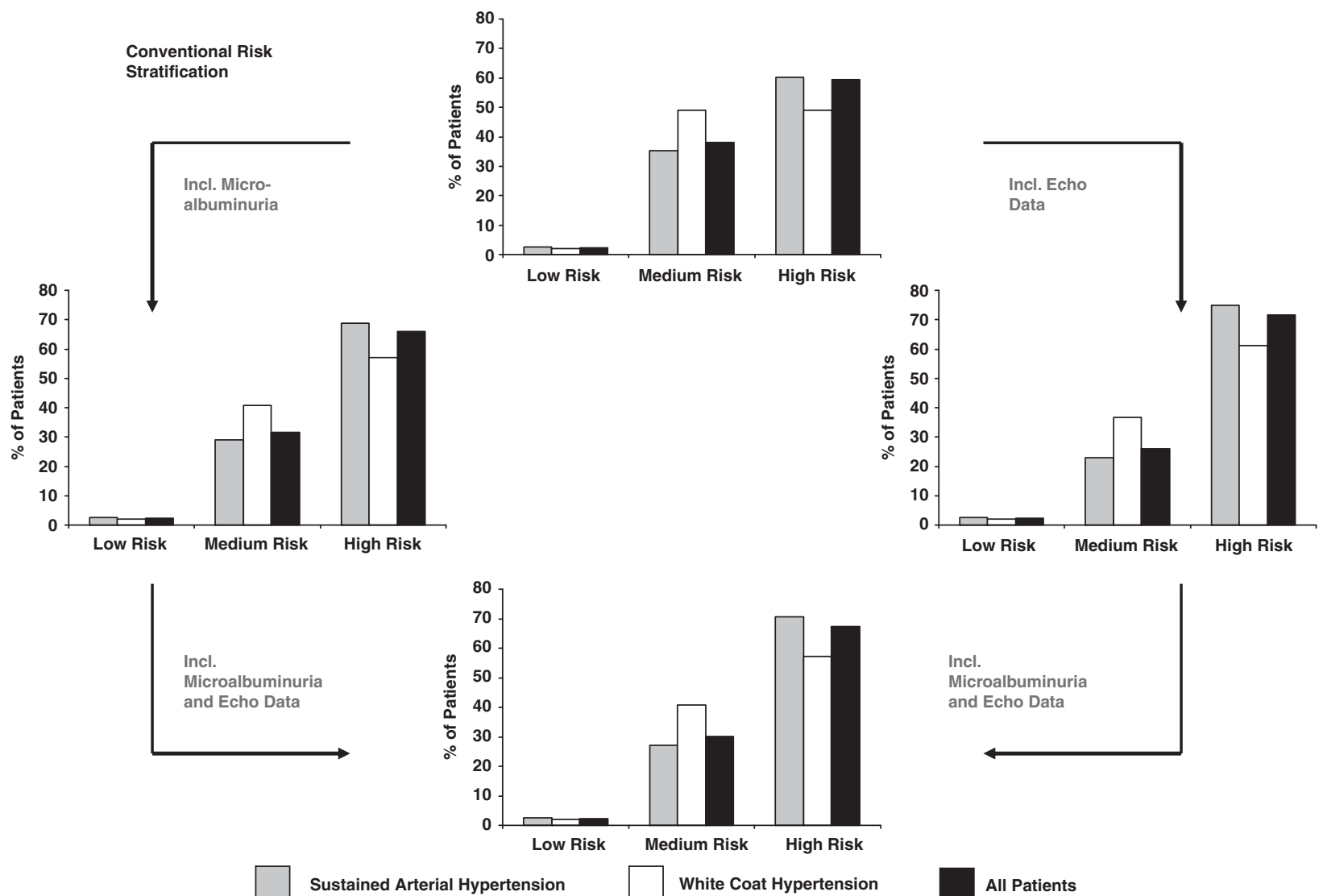

Figure 3 Distribution of absolute cardiovascular risk and contribution of diagnostic procedures to risk stratification in patients with sustained and white coat hypertension.

Table 3 Contribution of office blood pressure, risk factor status, evidence of target organ damage or associated clinical conditions to categorization as high-risk patients

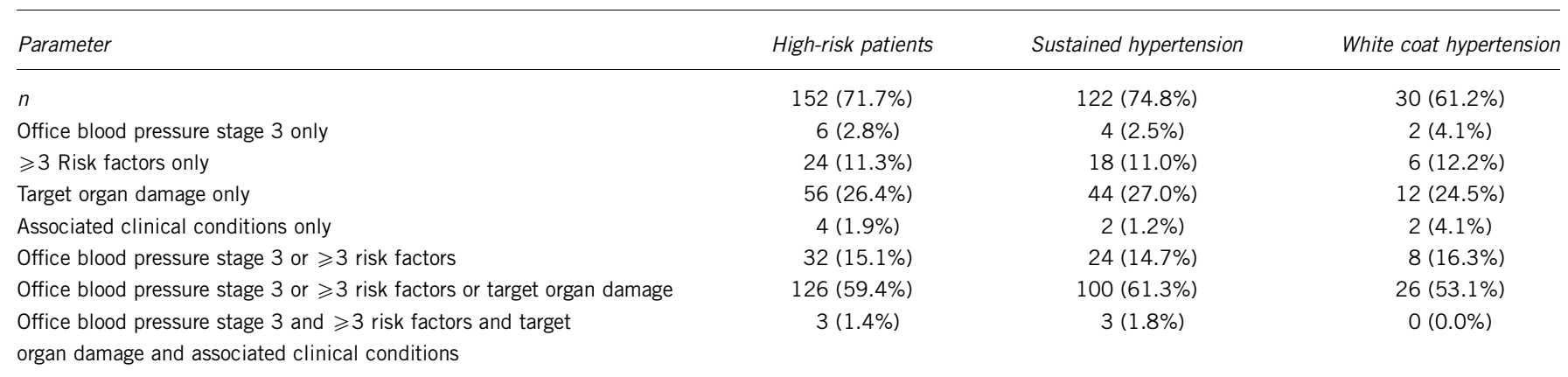

The number of high-risk patients. The percentage of all patients is shown in parenthesis.

High prevalence of undetected arterial hypertension in a general medical patient population

The prevalence of arterial hypertension in the study population was considerably lower than that recently reported by the WHO-Monica project. ${ }^{29}$ Awareness rates for arterial hypertension were reported to be $53-68 \%$ in Switzerland. ${ }^{29}$ Thus, given the dense primary health-care network in Switzerland, it was surprising that almost $10 \%$ of the study population had newly detected arterial hypertension according to the standard guidelines. ${ }^{1,3}$ It is important to note that these patients entered the Medical Outpatient Department because of problems not related to arterial hypertension, with many of them as walk-in patients who do not regularly use other primary care facilities. However, this finding underlines the importance of screening every patient for arterial hypertension, as it is the most important risk factor for cardiovascular morbidity and mortality.

High CVR in newly detected hypertensive patients

The most important finding of our study was that the majority of unselected patients with newly detected arterial hypertension in a general medical setting were at high CVR. Risk stratification using medical history and clinical and routine laboratory exams identified approximately $59 \%$ of the patients as being at high risk. 
Table 4 Characteristics of patients with elevated first and normal second office blood pressure values

\begin{tabular}{|c|c|}
\hline Parameter & \\
\hline$n$ & 36 \\
\hline Age (years) & $50.8 \pm 13.6^{*}$ \\
\hline Sex (female/male, $n$ ) & $52.8 \%(19) / 47.2 \%(17)$ \\
\hline Body mass index $\left(\mathrm{kg} \mathrm{m}^{-2}\right)$ & $27.2 \pm 4.6$ \\
\hline \multicolumn{2}{|c|}{ Office blood pressure ( $\mathrm{mm} \mathrm{Hg}$ ) } \\
\hline Visit 1 (SBP/DBP) & $155 \pm 11 * / 100 \pm 3$ \\
\hline Visit 2 (SBP/DBP) & $128 \pm 8^{*} / 82 \pm 6^{*}$ \\
\hline \multicolumn{2}{|c|}{ Ambulatory blood pressure $(\mathrm{mm} \mathrm{Hg})$} \\
\hline $24 \mathrm{~h}(\mathrm{SBP} / \mathrm{DBP})$ & $123 \pm 8^{*} / 77 \pm 6^{*}$ \\
\hline Day (SBP/DBP) & $127 \pm 8 * / 81 \pm 7 *$ \\
\hline Night (SBP/DBP) & $117 \pm 10 * / 72 \pm 6^{*}$ \\
\hline Sustained hypertension & $33.3 \%$ \\
\hline
\end{tabular}

Abbreviations: DBP, diastolic blood pressure $(\mathrm{mm} \mathrm{Hg})$; SBP, systolic blood pressure $(\mathrm{mm} \mathrm{Hg})$ ${ }^{*} P<0.05$ vs. patients with elevated first and second office blood pressure values.

After inclusion of data on microalbuminuria or echocardiographic LVH for risk stratification, 66 and $67.5 \%$ of patients, respectively, were classified as being at high CVR. Compared with routine risk stratification, the inclusion of both microalbuminuria and echocardiographic LVH very significantly increased the rate of patients at high CVR (to $72 \%$ ), identifying an additional $13 \%$ of patients previously classified as being at low or medium risk.

Several studies have analyzed the effect of microalbuminuria and echocardiographic results on risk stratification in hypertensive patients. However, these studies were restricted to pre-selected patient groups, such as patients with mild ${ }^{17,30}$ or moderate hypertension ${ }^{19}$ or patients admitted to hypertension clinics. ${ }^{31,32}$

Most studies reported relatively low rates of patients with high CVR when routine methods for risk stratification were applied. This rate increased substantially when microalbuminuria or ultrasound techniques such as echocardiography and carotid ultrasound were also taken into account. ${ }^{4,32,33}$

In contrast to these previous studies, our study shows a relatively high percentage of patients at high CVR after routine evaluation. At this stage of the diagnostic process, classification as being at high risk was due to diabetes mellitus and ACCs and-most important- to the high rate of patients with three or more CVRFs besides diabetes. Besides routine risk factor assessment and treatment of ACCs, our study again shows the importance of assessing TOD in the hypertensive population. Together with data from previous studies, the results of our study emphasize the need to take all diagnostic modalities into account to obtain a reliable estimate of CVR for an individual patient.

As mentioned above, the most prevalent risk factors were lack of physical activity, family history of cardiovascular disease and smoking. Therefore, addressing and aggressively treating the modifiable risk factors in the hypertensive population will have a large effect on overall CVR, emphasizing the importance of a holistic approach to the patient with arterial hypertension.

\section{The use of echocardiography for risk stratification}

As in other studies, our data show that echocardiography is especially useful for risk stratification in patients previously classified as being at low or medium CVR. ${ }^{17,19}$ Echocardiography identified an additional 12 of 72 patients (16.6\%) at high CVR who were classified as being at low or medium risk after conventional risk stratification and urinalysis. This finding indicates that all patients at low or medium CVR after assessment of past medical history, physical status, routine laboratory analysis and urinalysis-procedures that can easily be performed in any medical office-should undergo echocardiographic evaluation for LVH.

\section{White coat hypertension and overall CVR}

The most surprising finding of our study was that no difference was found between white coat and sustained hypertensives regarding the proportion of patients at low, medium and high CVR. According to risk stratification using OBP measurements, information from medical history, clinical status, laboratory assessment, ECG, microalbuminuria and echocardiography, most of the patients with white coat hypertension were at high CVR.

There is continuing debate over whether white coat hypertension is an innocent phenomenon or whether it carries an increased burden of CVR. ${ }^{34,35}$ From our data, we conclude that white coat hypertension is not negligible in most patients and that thorough clinical evaluation is needed to exclude TOD. Currently, guidelines do not generally recommend antihypertensive treatment in patients with white coat hypertension. However, in view of our data, a therapy decision based on the individual risk profile and the presence of TOD seems to be justified. At any rate, close follow-up is essential for subjects whom the physician chooses not to treat. Our results also emphasize the need for further studies analyzing CVR and the potential benefits of drug therapy targeted to end-organ damage in patients with white coat hypertension.

\section{CONCLUSIONS}

In summary, we have found a high rate of CVRFs, TOD and, consequently, a high overall CVR in unselected medical outpatients with newly detected arterial hypertension. Our study shows that all diagnostic modalities, including ECG, echocardiography and urinalysis, must be applied to correctly classify CVR in this patient population. In view of a majority of patients at high risk for cardiovascular events, a 'wait-and-watch' strategy may be justified only after exclusion of high CVR in an individual patient.

No significant differences regarding the number and distribution of CVRFs or the prevalence of TOD were found between sustained and white coat hypertensives, indicating a substantially greater CVR in the latter group. In summary, the data of this study underline the importance of a holistic approach to the hypertensive patient that addresses concomitant risk factors as well as TOD and existing ACCs.

\section{CONFLICT OF INTEREST}

The authors declare no conflict of interest.

\section{ACKNOWLEDGEMENTS}

This study was supported by an unrestricted grant from Novartis Pharma AG, Switzerland.

1 Whitworth JA, World Health Organization, International Society of Hypertension Writing Group. 2003 World Health Organization (WHO)/International Society of Hypertension (ISH) statement on management of hypertension. J Hypertens 2003; 21 : 1983-1992.

2 Lever AF, Ramsay LE. Treatment of hypertension in the elderly. J Hypertens 1995; 13: 571-579.

31999 World Health Organization-International Society of Hypertension Guidelines for the Management of Hypertension. Guidelines Subcommittee. J Hypertens 1999; 17: 151-183. 
4 Viazzi F, Leoncini G, Parodi D, Ratto E, Vettoretti S, Vaccaro V, Parodi A, Falqui V, Tomolillo C, Deferrati G, Pontremoli R. Impact of target organ damage assessment in the evaluation of global risk in patients with essential hypertension. J Am Soc Nephrol 2005; 16: S89-S91.

5 Cuspidi C, Meani S, Valerio C, Fusi V, Sala C, Zanchetti A. Left ventricular hypertrophy and cardiovascular risk stratification: impact and cost-effectiveness of echocardiography in recently diagnosed essential hypertensives. J Hypertens 2006; 24: 1671-1677.

6 Coca A, Gabriel R, de la Figuera M, Fernandez R, Sagastagoitia JD, Garcia JJ, Barajas $\mathrm{R}$. The impact of different echocardiographic diagnostic criteria on the prevalence of left ventricular hypertrophy in essential hypertension: the VITAE study. J Hypertens 1999; 17: 1471-1480.

7 Martina B, Dieterle T, Weinbacher M, Battegay E. Effects of losartan titrated to losartan/ hydrochlorothiazide and amlodipine on left ventricular mass in patients with mild-tomoderate hypertension. Cardiology 1999; 92: 110-114.

8 Tingleff J, Munch M, Jakobsen TJ, Torp-Pedersen C, Olsen ME, Jensen KH, Jorgensen T, Kirchoff M. Prevalence of left ventricular hypertrophy in a hypertensive population. Eur Heart J 1996; 17: 143-149.

9 Gottdiener JS, Reda DJ, Materson BJ, Notargiacomo A, Hamburger RJ, Williams DW, Henderson WG. Importance of obesity, race and age to the cardiac structural and functional effects of hypertension. The Department of Veterans Affairs Cooperative Study Group on antihypertensive agents. J Am Coll Cardiol 1994; 24: 1492-1498.

10 Hammond IW, Devereux RB, Alderman MH, Lutas EM, Spitzer MC, Crowley JS, Laragh $\mathrm{JH}$. The prevalence and correlates of echocardiographic left ventricular hypertrophy among employed patients with uncomplicated hypertension. J Am Coll Cardiol 1986; 7: 639-650.

11 Fragola PV, Colivicchi F, Fabrizi E, Borzi M, Cannata D. Assessment of left ventricular hypertrophy in patients with essential hypertension. A rational basis for the electrocardiogram. Am J Hypertens 1993; 6: 164-169.

12 Verdecchia P, Dovellini EV, Gorini M, Gozzelino G, Lucci D, Milletich A, Maggioni AP. Comparison of electrocardiographic criteria for diagnosis of left ventricular hypertrophy in hypertension: the MAVI study. Ital Heart J 2000; 1: 207-215.

13 Cesarola G, Cottone S, D'Ignoto G, Grasso L, Mangano MT, Carapelle E, Nardi E, Andronico G, Fulantelli MA, Marcellino T. Microalbuminuria as a predictor of cardiovascular damage in essential hypertension. J Hypertens 1989; 7(Suppl): 332-333.

14 Pontremoli R, Sofia A, Ravera M, Nicolella C, Viazzi F, Tirotta A, Ruello N, Tomolillo C, Castello C, Grillo G, Sacchi G, Deferrari G. Prevalence and clinical correlates of microalbuminuria in essential hypertension. The MAGIC study. Hypertension 1997; 30: 1135-1143.

15 Wachtell K, Olsen MH, Dahlöf B, Devereux RB, Kjeldsen SE, Nieminen MS, Okin PM, Papademetriou V, Mogensen CE, Borch-Johnsen K, Ibsen $\mathrm{H}$. Microalbuminuria in hypertensive patients with electrocardiographic left ventricular hypertrophy: the LIFE study. J Hypertens 2002; 20: 405-412.

16 Donnelly R, Rea R. Microalbuminuria: how informative and reliable are individual measurements? J Hypertens 2003; 21: 1929-1933.

17 Martinez MA, Sancho T, Armada E, Rubio JM, Antón JL, Torre A, Palau J, Seguido P, Gallo J, Saenz I, Polo E, Torres R, Oliver J, Puig JG, on behalf of the Vascular Risk Working Group “Grupo de Monitorizaci ó n Ambulatoria de la Presi ó n Arterial (MAPA)Madrid'. Prevalence of left ventricular hypertrophy in patients with mild hypertension in primary care: impact of echocardiography on cardiovascular risk stratification. Am J Hypertens 2003; 16: 556-563.

18 Schillaci G, de Simone G, Reboldi G, Porcellati C, Devereux RB, Verdecchia P. Change in cardiovascular risk profile by echocardiography in low- or medium-risk hypertension. J Hypertens 2002; 20: 1519-1525.

19 Cuspidi C, Michev L, Severgnini B, Meani S, Fusi V, Valerio C, Bertazzoli G, Magrini F, Zanchetti A. Change in cardiovascular risk profile by echocardiography in medium-risk elderly hypertensives. J Hum Hypertens 2003; 17: 101-106.
20 Cesarola G, Mulè G, Nardi E, Cottone S, Andronico G, Mongiovi R, Mezzatesta G. Usefulness of microalbuminuria in cardiovascular risk stratification of essential hypertensive patients. Nephron Clin Pract 2004; 96: c123-c130.

21 Clement DL, De Buyzere ML, De Bacquer DA, de Leeuw PW, Duprez DA, Fagard RH, Gheeraert PJ, Missault LH, Braun JJ, Six RO, Van Der Niepen P, O'Brien E, Office versus Ambulatory Pressure Study Investigators. Prognostic value of ambulatory bloodpressure recordings in patients with treated hypertension. N Engl J Med 2003; 348: 2407-2415.

22 Verdecchia P. Prognostic value of ambulatory blood pressure: current evidence and clinical implications. Hypertension 2000; 35: 844-851.

23 Mule G, Caimi G, Cottone S, Nardi E, Andronico G, Piazza G, Volpe V, Federico MR, Cerasola G. Value of home blood pressures as predictor of target organ damage in mild arterial hypertension. J Cardiovasc Risk 2002; 9: 123-129.

24 Bennett PH, Haffner S, Kasiske BL, Keane WF, Mogensen CE, Parving HH, Steffes MW, Striker GE. Screening and management of microalbuminuria in patients with diabetes mellitus: recommendations of the Scientific Advisory Board of the National Kidney Foundation from an ad hoc committee of the Council on Diabetes Mellitus of the National Kidney Foundation. Am J Kidney Dis 1995; 25: 107-112.

25 Sokolow M, Lyon TP. The ventricular complex in left ventricular hypertrophy as obtained by unipolar precordial and limb leads. Am Heart J 1949; 37: 161-186.

26 Molloy TJ, Okin PM, Devereux RB, Kligfield P. Electrocardiographic detection of left ventricular hypertrophy by the simple QRS voltage duration product. J Am Coll Cardiol 1992; 20: 1180-1186.

27 Sahn DJ, DeMaria A, Kisslo J, Weyman A. Recommendations regarding quantitation in M-mode echocardiography: results of a survey of echocardiographic measurements. Circulation 1978; 58: 1072-1083.

28 Devereux RB. Detection of left ventricular hypertrophy by M-mode echocardiography. Anatomic validation, standardization, and comparison to other methods. Hypertension 1987; 9(2 Pt 2): II19-II26.

29 Antikainen RL, Moltchanov VA, Chukwuma Sr C, Kuulasmaa KA, Marques-Vidal PM, Sans S, Wilhelmsen L, Tuomilehto JO, WHO MONICA Project. Trends in the prevalence, awareness, treatment and control of hypertension: the WHO MONICA Project. Eur J Cardiovasc Prev Rehabil 2006; 13: 13-29.

30 Martinez MA, Moreno A, Aguirre de Carcer A, Cabrera R, Rocha R, Torre A, Nevado A, Ramos T, Neri J, Anton G, Miranda I, Fernandez P, Rodriguez E, Miquel A, Martinez JL, Rodriguez M, Eisman C, Puig JG, MAPA- Madrid Working Group. Frequency and determinants of microalbuminuria in mild hypertension: a primary-care-based study. J Hypertens 2001; 19: 319-326.

31 Cuspidi C, Lonati L, Macca G, Sampieri L, Fusi V, Severgnini B, Salerno M, Iassen M, Rocanova JI, Leonetti G, Zanchetti A. Cardiovascular risk stratification in hypertensive patients: impact of echocardiography and carotid ultrasonography. J Hypertens 2001; 19: 375-380.

32 Leoncini G, Sacchi G, Viazzi F, Ravera M, Parodi D, Ratto E, Vettoretti S, Tomolillo C, Deferrari G, Pontremoli R. Microalbuminuria identifies overall cardiovascular risk in essential hypertension: an artificial neural network-based approach. J Hypertens 2002; 20: 1315-1321.

33 Cuspidi C, Ambrosioni E, Mancia G, Pessina AC, Trimarco B, Zanchetti A, On behalf of the APROS investigators. Role of echocardiographic and carotid ultrasonography in stratifying risk in patients with essential hypertension: the Assessment of Prognostic Risk Observational Survey. J Hypertens 2002; 20: 1307-1314.

34 Angeli F, Verdecchia P, Gattobigio R, Sardone M, Reboldi G. White-coat hypertension in adults. Blood Press Monit 2005; 10: 301-305.

35 Ohkubo T, Kikuya M, Metoki H, Asayama K, Obara T, Hashimoto J, Totsune K, Hoshi H, Satoh H, Imai Y. Prognosis of 'masked' hypertension and 'white-coat' hypertension detected by 24-h ambulatory blood pressure monitoring 10-year follow-up from the Ohasama study. J Am Coll Cardiol 2005; 46: 508-515. 